\title{
Non-Conventional Applications of Computerized Tomography: Analysis of Solid Dosage Forms Produced by Pharmaceutical Industry
}

\author{
José Martins de Oliveira Jr. ${ }^{a}$ and Antonio César Germano Martins ${ }^{b}$ \\ ${ }^{a}$ Universidade de Sorocaba - UNISO, Campus Trujillo, Caixa Postal 578, \\ Av. Dr. General Osório, 35, Centro, 18060-000, Sorocaba, SP, Brazil \\ ${ }^{b}$ Universidade Estadual Paulista Julio de Mesquita Filho - UNESP, GASI, Av. 3 de Março, 511, Alto da \\ Boa Vista, 18087-180, Sorocaba, SP, Brazil.
}

\begin{abstract}
X-ray computed tomography (CT) refers to the cross-sectional imaging of an object measuring the transmitted radiation at different directions. In this work, we describe a nonconventional application of computerized tomography: visualization and improvements in the understanding of some internal structural features of solid dosage forms. A micro-CT X-ray scanner, with a minimum resolution of $30 \mu \mathrm{m}$ was used to characterize some pharmaceutical tablets, granules, controlled-release osmotic tablet and liquid-filled soft-gelatin capsules. The analysis presented in this work are essentially qualitative, but quantitative parameters, such as porosity, density distribution, tablets dimensions, etc. could also be obtained using the related $\mathrm{CT}$ techniques.
\end{abstract}

Keywords: X-ray, Computed Tomography, Tablets, Granules, Solid Dosage Forms.

PACS: 81.70.Tx; 82.80.Ej; 87.59.-e

\section{INTRODUCTION}

X-ray computed tomography (CT) is a nondestructive technique used to obtain images of internal structure of a body. The fundamental principle behind CT, or image reconstruction from projections, has been known since the studies made by Radon [1] in 1917, in which the Radon inversion formula was derived and proved. CT methods have been used in many areas such as: soil science [2], study of porous structure of amorphous materials [3], etc. In recent years, many non-conventional uses of CT has been introduced. Some of theses, for instance, applied to pharmaceutical industry, are: a) use of X-ray tomography to study the porosity and morphology of granules [4], b) measurement of density variation in tablets [5] and c) studies of internal structures of solid dosage forms [6]. This new approach to study properties of tablets, powders, granulations and liquid filled gelatin capsule is very suitable, first, because CT could generate information that traditional technologies used in this kind of analysis could not, such as: density distribution of internal structures without destroying the sample, tablet dimensions and integrity, pore size distribution, particle shape information, 
investigation of official and unofficial (counterfeit) copies of solid dosage forms and, second, because CT is a nondestructive technique, allowing the use of solid dosage forms in others analysis and also requires no specimen preparation. CT is a technique that is based on attenuation of X-rays passing through matter. This work shows and discusses how to obtain qualitative information of internal and external structure from pharmaceutical forms based on non-conventional tomographic images of tablets, granules, gelatin capsules and special controlled-release tablet.

\section{MATERIALS AND METHODS}

$\mathrm{X}$-ray computed tomography is a nondestructive inspection technique which provides cross-sectional 2D images in planes through of the knowledge of projections. Mathematical algorithms for tomographic reconstruction are based on projection data. These projections represent the linear attenuation coefficients of X-rays, along the ray path. The attenuation coefficients are dependent on the photon energy as well as on the density and the atomic number of objects or their constituents. The most widely used reconstruction technique is called Filtered Back Projection (FBP). Details on the theory of image reconstruction and practical algorithms may be found, for instance, in Ref. [7].

The micro-CT scanner used in this work is a third generation type and is located at the Applied Nuclear Physics Laboratory of University of Sorocaba (LAFINAU) in Sorocaba, SP, Brazil [8]. Data were acquired using different pharmaceutical solid dosage forms, with the following X-ray CT facility setup: a) 35 $\mathrm{keV}$ to $50 \mathrm{keV}$ of X-ray energy range; b) $50 \mu \mathrm{A}$ to $70 \mu \mathrm{A}$ intensity filament current of $\mathrm{X}$-ray source; c) 500 to 1000 projection data per image and d) $30 \mu \mathrm{m}$ to $100 \mu \mathrm{m}$ of final image resolution, depending the configuration choice to perform the acquisition.

\subsection{X-ray Source}

X-ray CT facility uses a microfocus X-ray source from Hamamatsu Corp. model L6731-01. This X-ray source has a focal spot size of $8 \mu \mathrm{m}$ and produces a fan beam with an aperture angle of 39 degrees. This configuration is very appropriate to obtain image with high magnification and resolution. The X-ray source operates in a continuous mode and generates X-rays with high stability, with fluctuations no larger than $0.2 \%$ measured after two hours of operation. The target source is made of tungsten and operates with voltage between $20 \mathrm{KV}$ to $80 \mathrm{KV}$ and can supply X-rays with variable intensity by modifying the target current from $0 \mu \mathrm{A}$ to $100 \mu \mathrm{A}$.

\subsection{Radiation Detector}

X-ray CT facility uses one commercial photodiode array (APD) from Hamamatsu Corp. model S8865-128G. This APD has $300 \mu \mathrm{m}$ x $600 \mu \mathrm{m}$ of dimensions and a tin layer of phosphor deposited at the entrance, becoming this array sensitive to X-rays in the energy range from $30 \mathrm{KeV}$ to $100 \mathrm{KeV}$. This APD has an appropriate spatial resolution for the proposed applications, but it does not have any energy discrimination. 


\subsection{Sample Positioning and Data Acquisition}

Step motors are used to sample positioning. No beam collimator has beam used in front of the detectors, due to the difficult in producing a collimator for the PDA dimensions. The correct positioning and alignment of all movables parts are essential for obtaining good tomography images. The distance source-object-detector is dependent of object size and resolution required in the tomography. The object and detectors can be moved to achieve the better configuration for each purpose. At the Xray $\mathrm{CT}$ facility, the horizontal source-object-detector distance can vary in a range from $12 \mathrm{~mm}$ to $400 \mathrm{~mm}$. The sample rotates in a horizontal plane and can also be vertically translated (from $0 \mathrm{~mm}$ to $300 \mathrm{~mm}$ ) by two step motor. Each measure from all detectors for a given object position is usually denominate a projection. One the most important feature of a third-generation scanner is the number of projections used to form an image. The number of angles per rotation used in each tomography, in the X-ray CT facility, can be chosen by the user. Once the object is placed on the rotational table, the tomography is started. To control all equipments and data acquisition, a high-speed multifunction M-series data acquisition (DAQ) PCI-6251 from National Instruments Corp. is used. This DAQ has 16-Bit of accuracy and can work with sampling rates as far as $1.25 \mathrm{MS} /$ Channel. The DAQ has several analog and digital input and output allowing a precise control of all process and equipment involved in a tomography. A man-machine interface is based on NI LabVIEW software and was developed to control all process evolved in tomograph operation.

\subsection{Image Reconstruction}

The mathematical algorithms for tomographic reconstruction are based on projection data. These projections can represent, for example, the linear attenuation coefficients of X-rays, along the path of the ray. The attenuation coefficients are energy dependent of photons, as well as the density of objects or their constituents along the ray path. For the implemented solution, X-ray is considered an energy independent function. The Beer's Law, that is the basic equation for attenuation of a monoenergetic narrow beam, can be written as:

$$
P(r, \theta)=\int_{-\infty}^{+\infty} \mu(x, y) d l=\ln \left(\frac{I_{0}}{I}\right),
$$

where the projection ray sums $P(r, \theta)$ are expressed in polar coordinates as a function of distance $r$ from the origin and angle $\theta$ relative to the $\mathrm{x}$-axis of the cartesian coordinate reconstruction plane, $\mu(x, y)$ is a function of position within the reconstruction plane whose magnitude evaluate at any point $(x, y)$ is the linear attenuation coefficient at the point, and $d l$ is the differential path length along any ray. $I_{0}$ and $I$ are the incident and output photons flux intensity (photons/unit time and area) measured by PDAs. The basic problem in tomography is to invert Eq. 1 to solve for $\mu(x, y)$ through the section of the sample crossed by radiation. The most widely used reconstruction technique is called the convolution method, or Filtered Back Projection (FBP) method. In this method, data are first convolved with a filter and each filtered 
projection data is successively superimposed over a square grid at an angle corresponding to its acquisition angle, or back-projected. In the spatial domain filtering step may be expressed as the convolution:

$$
P^{*}(r, \theta)=\int_{-\infty}^{+\infty} P\left(r^{\prime}, \theta\right) \cdot h\left(r-r^{\prime}\right) d r^{\prime},
$$

where $P^{*}$ denotes convolved projection, and $h(r)$ is called the convolution Kernel. The value of $\mu(x, y)$ can be obtained integrating the filtered projections over all projection angles:

$$
\mu(x, y)=\int_{0}^{\pi} P^{*}(x \cos \theta+y \sin \theta, \theta) d \theta .
$$

In practice some assumptions are made in order to reduce the amount of information and simplify the computational work. Eqs. 2 and 3 may be numerically implemented by replacing the integrals with summations. More details on the theory of image reconstruction and practical algorithms may be found, for instance, in Ref. [7].

\section{RESULTS AND DISCUSSION}

Tomographic images showed in this section were corrected from detector imperfection output and from some artifacts that are common in this type of tomographic system, such as: ring artifact and beam hardening. Other important correction used to improve image quality, consists in taking a tomographic image of an empty field. Object image is then subtracted from this empty image to resume the final image. The reasons for this correction are that: i) X-ray detectors are uneven in intensity and often more sensitive towards the centre and, ii) X-ray beam is also nonuniform, often being stronger in the centre [5]. The final images are obtained in gray scale, but it also could be manipulated by using software techniques to produce binary or multiple-colored images. Figure 1, part (1)-(a), shows a CT image of an experimental dog's tablet with a bone shape and used to treat dog's arthritis. The dimensions of these dog's tablets are $25 \mathrm{~mm} \times 12 \mathrm{~mm}$ approximately. X-ray CT facility setup used to produce the image showed in Figure 1, part (1)-(a) was: $50 \mathrm{keV}$ of X-ray energy; $40 \mu \mathrm{A}$ of intensity filament current of X-ray source; 500 projection data (angular step equal 0.72 degrees) and $100 \mu \mathrm{m}$ of resolution. Analyzing this image, many color tonality can be discriminated, indicating the capability of the technique in resolving different materials, or densities, inside the tablet. Probably, blue areas showed in Figure 1 part (1)-(a) are inhomogeneous distribution of an active ingredient used in tablet formulation. Figure 1, part (1)-(b), it is shown a photography of the tablet. Figure 1, part (2)-(a), shows a typical CT cross-sectional image of the granules inside the capsule. These granules, about $1 \mathrm{~mm}$ of diameter each, are microcrystalline cellulose. In Figure 1, part (2)-(a) it is possible to see how granules are accommodate inside de capsule and in part (2)-(b) an expanded portion of these 
granules is showed. In this amplified image, the pore structure of a granule is revealed showing some voids inside the granules. This image was made using $35 \mathrm{keV}$ of X-ray energy; $70 \mu \mathrm{A}$ of intensity filament current of X-ray source; 1000 (angular step equal 0.36 degrees) projection data and $36 \mu \mathrm{m}$ of resolution. This image indicates a good possibility to use CT techniques in studying the morphology and pore-size distribution of pharmaceutical granules.

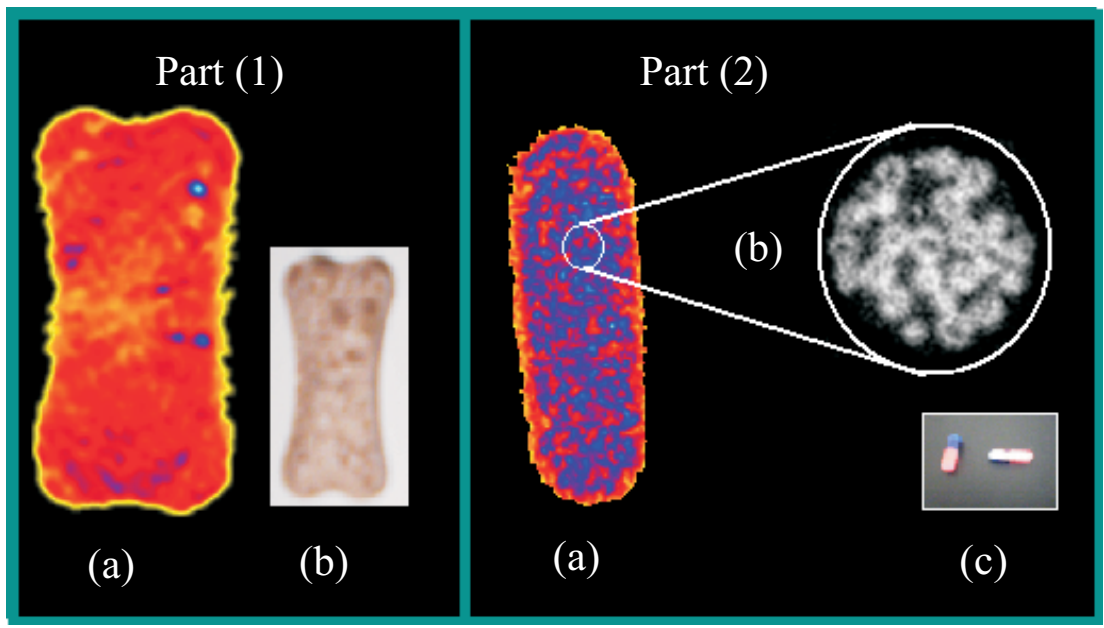

FIGURE 1. Part (1)-(a), shows a tomographic image of an experimental dog's tablet, having a bone shape. The dimensions of these dog's tablets are $25 \mathrm{~mm} \times 12 \mathrm{~mm}$ approximately. The blue areas showed in part (1)-(a) are inhomogeneous distribution of tablets constituents and (b) shows a photography image. Part (2)-(a), shows a tomographic image of an experimental capsule fulfill with microcrystalline cellulose. Part (2)-(b) shows an expanded image of these granules and (c) is a photography image of this capsule. The tomographic images were reconstructed using FBP method.

Figure 2, part (1)-(a), shows a tomographic image of an osmotic controlledreleased dosage form, used by people that need arterial pressure control. Its functional principle is based on expansion of a crystal layer, with about $1.5 \mathrm{~mm}$ of thickness, when some quantity of water or gastric juice penetrates the tablet. This expands the crystal layer and expulses the drug by an orifice located at the opposite side of the tablet. With this CT image, it is possible to obtain the key tablet dimensions. These data also indicates that CT techniques could be used to perform dynamic studies, which take place inside this type of medicament, as water penetrates then. Figure 2, part (2)-(a), shows a tomographic image of a commercial gelatin capsule, filled with a liquid substance containing an anti-inflammatory drug. In this type of capsule, it is very important to guarantee film-coating integrity to avoid substance leakage. The analyzed tomographic image shows a perfect film-coating of soft-gelatin capsule, which about $0,4 \mathrm{~mm}$ of thickness. Both images showed in Figure 2 were made using $50 \mathrm{keV}$ of X-ray energy; $50 \mu \mathrm{A}$ of intensity filament current of X-ray source; 1000 projection (angular step equal 0.36 degrees) data and $100 \mu \mathrm{m}$ of resolution. 


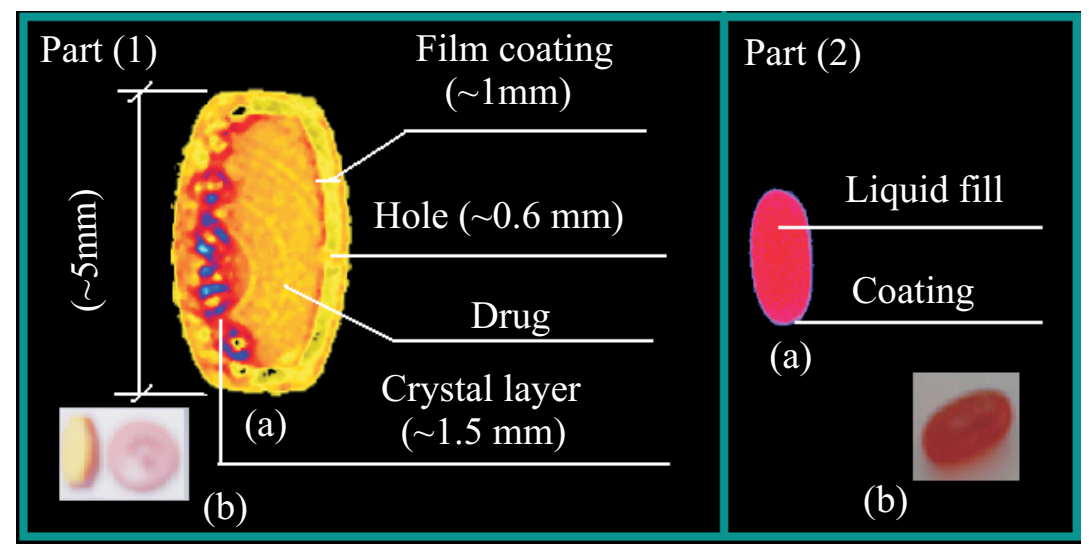

FIGURE 2. Part (1)-(a), shows a tomographic image of a commercial osmotic controlled-released tablet. The Part (1)-(a) shows yet, some structure of this tablet, such as: key tablet dimensions, diameter of a hole in the coating and thickness of crystal layer. Part (1)-(b) is a photography image. Part (2)-(a) shows a tomographic image of a commercial gelatin capsule, fulfill with a liquid substance. Part (2)-(b) is a photography image of this capsule. The tomographic images were reconstructed using FBP method.

\section{FINAL CONSIDERATIONS}

It is discussed a non-conventional use of CT applied in the study of solid dosage forms (tablets, capsules and granules). The main purpose of this work was to shown the many possibilities of CT use to perform qualitative and/or quantitative analysis of pharmaceutical solid dosage form. Important features involved in the formulation and in the behavior of these dosage forms are discussed. Tomographic images shown a non uniform density distribution of constituents in the experimental dog tablet, the morphology of a granules used in the fulfillment of capsules, the integrity liquid-filled soft-gelatin capsule and the key dimensions of an osmotic controlled-release dosage form. We believe that in the future, studies involving timedependent process of drug liberation and water movement inside the tablet will be available with CT techniques.

\section{ACKNOWLEDGMENTS}

The authors would like to thank the Fundação de Amparo à Pesquisa do Estado de São Paulo (FAPESP), Brazil, Grants 05/04727-6.

\section{REFERENCES}

1. J. Radon, "In the determination of functions from their integrals along certain manifolds", Berchte über die Verhandlugen, 69, 262-277 (1917).

2. P. E. Cruvinel, R. Cesareo, S. Crestana and S. Mascarenhas, "X-ray and gamma-ray computerized minitomograph scanner for soil science”, IEEE Trans. Instrum. Meas., 39, (5), 745-750 (1990). 
3. C. R. Appoloni, C. P. Fernandes, M. D. M. Innocentini, A. Macedo, "Ceramic Foams Porous Microstructure Characterization By X-ray Microtomography”, Mat. Res., 7, (4), 557-564 (2004).

4. L. Farber, G. Tardos, J. N. Michaels, "Use of X-ray tomography to study the porosity and morphology of granules", Powder Technology 132, 57-63 (2003).

5. I. C. Sinka, S. F. Burch, J. H. Tweed, J. C. Cunningham, "Measurement of density variations in tablets using X-ray computed tomography", International Journal of Pharmaceutics. 271, 215-224 (2004).

6. B. C. Hancock, M. P. Mullarney, "X-ray Microtomography of Solid Dosage Forms", Pharmaceutical Technology, 92-100 (2005).

7. A. Kak and M. Slaney, "Principles of Computerized Tomographic Imaging", IEEE Press, New York, (1988).

8. J. M. Oliveira Jr., A. C. G. Martins, "Construction and Test of Low Cost X-Ray Tomography Scanner for Physical-Chemical Analysis and Nondestructive Inspections", American Institute of Physics Conference Proceedings, 1139 (1), 102-105, (2009). 
Copyright of AIP Conference Proceedings is the property of American Institute of Physics and its content may not be copied or emailed to multiple sites or posted to a listserv without the copyright holder's express written permission. However, users may print, download, or email articles for individual use. 